\title{
MATRIX YOUNG NUMERICAL RADIUS INEQUALITIES
}

\section{Abbas Salemi And Alemeh SheikhHosseini}

Abstract. In the present paper, we show that if $A \in M_{n}(\mathbb{C})$ is a non scalar strictly positive matrix such that $1 \in \sigma(A)$, and $p>q>1$ with $\frac{1}{p}+\frac{1}{q}=1$, then there exists $X \in M_{n}(\mathbb{C})$ such that $\omega(A X A)>\omega\left(\frac{1}{p} A^{p} X+\frac{1}{q} X A^{q}\right)$. Moreover, several numerical radius inequalities are presented for Hilbert space operators. In particular, we prove that if $p \geqslant q>1$ with $\frac{1}{p}+\frac{1}{q}=1$, then $\omega^{r}\left(A^{*} X B\right) \leqslant\left\|\frac{1}{p}\left(A^{*}\left|X^{*}\right| A\right)^{\frac{r p}{2}}+\frac{1}{q}\left(B^{*}|X| B\right)^{\frac{r q}{2}}\right\|$, for all $A, B, X \in \mathscr{B}(\mathbf{H})$ and $r \geqslant \frac{2}{q}$.

Mathematics subject classification (2010): 15A60, 15A42, 47A30.

Keywords and phrases: Numerical radius inequality, Young inequality, norm, positive matrices.

\section{REFERENCES}

[1] T. Ando, Majorization and inequalities in matrix theory, Linear Algebra Appl. 199 (1994), 17-67.

[2] T. Ando, Matrix Young inequalities, Oper. Theory Adv. Appl. 75 (1995), 33-38.

[3] T. ANDO AND K. OKUBO, Induced norms of the Schur multiplication Operators, Linear Algebra and Appl. 147 (1991), 181-199.

[4] R. Bhatia, Positive Definite Matrices, Princeton University Press, 2007.

[5] R. Bhatia And F. KitTAneh, On the singular values of a product of operators, SIAM J. Matrix Anal. Appl. 11 (1990), 272-277.

[6] M. El-Haddad AND F. KitTANeH, Numerical radius inequalities for Hilbert space operators. II, Studia Mathematica 182, 2 (2007), 133-140.

[7] K.E. Gustafs On AND D.K.M. RaO, Numerical Range, Springer-Verlag, New York, 1997.

[8] F. KitTANEH, A numerical radius inequality and an estimate for the numerical radius of the Frobenius companion matrix, Studia Mathematica 158, 1 (2003), 11-17.

[9] F. Kittaneh, Notes on some inequalities for Hilbert space operators, Publications of the Research Institute for Mathematical Sciences 24, 2 (1988), 283-293.

[10] F. KitTAneH, Numerical radius inequalities for Hilbert space operators, Studia Mathematica 168, 1 (2005), 73-80.

[11] Kh. Shebrawi And H. Albadawi, Numerical Radius and Operator Norm Inequalities, J. of Inequal. and Appl. 2009 (2009), 1-11. 